ISSN 0021-8944, Journal of Applied Mechanics and Technical Physics, 2015, Vol. 56, No. 3, pp. 443-453. (C) Pleiades Publishing, Ltd., 2015.

Original Russian Text (c) M. Hemmat Esfe, M. Akbari, A. Karimipour.

\title{
MIXED CONVECTION IN A LID-DRIVEN CAVITY
}

\section{WITH AN INSIDE HOT OBSTACLE FILLED BY AN $\mathrm{Al}_{2} \mathrm{O}_{3}-$ WATER NANOFLUID}

\author{
M. Hemmat Esfe, M. Akbari, and A. Karimipour
}

UDC 536.2

\begin{abstract}
The present paper focuses on the problem of a mixed convection fluid flow and heat transfer of an $\mathrm{Al}_{2} \mathrm{O}_{3}$-water nanofluid with the thermal conductivity and effective viscosity dependent on temperature and nanoparticle concentration inside a lid-driven cavity having a hot rectangular obstacle. The governing equations are discretized by using the finite volume method, and the SIMPLE algorithm is employed to couple the velocity and pressure fields. By using the developed code, the effects of the Richardson number and the diameter and volume fraction of $\mathrm{Al}_{2} \mathrm{O}_{3}$ nanoparticles on the flow, thermal fields, and heat transfer inside the cavity are studied. The obtained results show that the average Nusselt number for the entire range of the solid volume fraction decreases with an increase in the Richardson number and the nanoparticle diameter. The results also clearly indicate that addition of $\mathrm{Al}_{2} \mathrm{O}_{3}$ nanoparticles produces a remarkable enhancement on heat transfer with respect to that of the pure fluid.
\end{abstract}

Keywords: nanofluid, solid volume fraction, heat transfer, nanoparticle diameter.

DOI: $10.1134 /$ S0021894415030141

\section{INTRODUCTION}

Nanofluids are created by suspending nanometer-sized particles (less than $100 \mathrm{~nm}$ ) in a pure fluid such as water, ethylene glycol, or propylene glycol. The first to coin the term "nanofluids" for these fluids with superior thermal properties was Choi [1]. The existence of metallic nanoparticles with a high thermal conductivity (e.g., copper, aluminum, silver, and titanium) increases the thermal conductivity of such mixtures, thus, enhancing their overall heat transfer capability [2]. In recent years, nanofluids have attracted attention as a new innovation of heat transfer fluids in building heating, various heat exchangers, and plant and automotive cooling applications, because of their excellent thermal performance. Various benefits of the application of nanofluids include improved heat transfer, heat transfer system size reduction, minimal clogging of microchannels, etc [1].

Numerous investigations were conducted on thermophysical properties of nanofluids (effective dynamic viscosity, thermal conductivity, etc.) and energy transport in nanofluids (see, e.g., [3-6]). Many theoretical, numerical, and experimental studies on the influence of nanoparticles on convective heat transfer were reported.

On the other hand, the fluid flow and heat transfer in a cavity filled by a pure fluid driven by buoyancy were studied extensively in the literature. Mixed convection (a kind of convection including both natural and forced convection) plays a significant role in many applications in industry and engineering. Lakes and reservoirs [7], food processing, crystal growth [8], electronic cooling devices, drying technologies [9], solar collectors [10], and float glass production [11] are among current applications.

Department of Mechanical Engineering, Najaf Abad Branch, Islamic Azad University, Isfahan, Iran; M.hemmatesfe@gmail.com; M.akbai1983@gmail.com; arashkarimipour@gmail.com. Translated from Prikladnaya Mekhanika i Tekhnicheskaya Fizika, Vol. 56, No. 3, pp. 116-127, May-June, 2015. Original article submitted December 24, 2012; revision submitted August 31, 2013. 


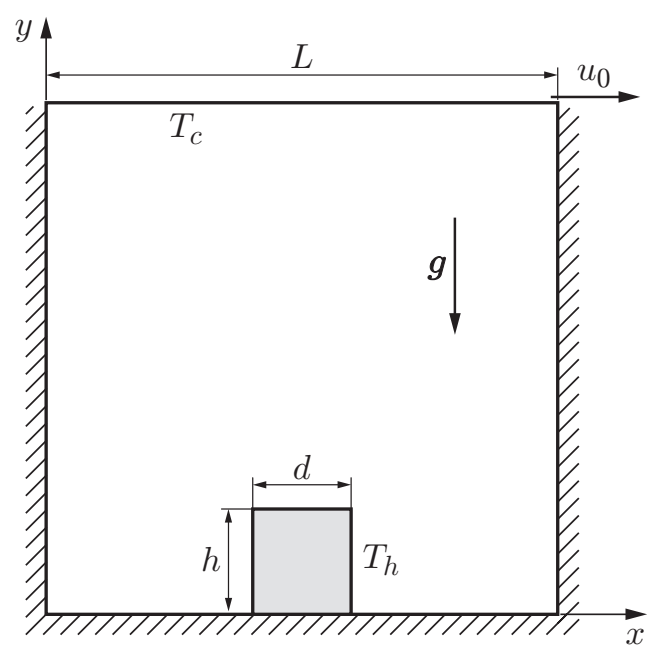

Fig. 1. Square cavity considered in this study.

Several investigations on mixed convection in a single or double lid-driven enclosure flow and heat transfer for different base fluids and boundary condition were reported [12-20].

Nikfar and Mahmoodi [21] studied natural convection in a square cavity filled by an $\mathrm{Al}_{2} \mathrm{O}_{3}$-water nanofluid. The horizontal walls of the cavity were insulated, while the left and right wavy side walls of the cavity were maintained at high and low constant temperatures. They demonstrated that an increase in the volume fraction of nanoparticles leads to an increase in the average Nusselt number on the hot wall.

A free convection fluid flow and heat transfer was recently investigated numerically by Mahmoodi and Mazrouei [22]. Their work included a $\mathrm{Cu}$-water nanofluid flow around adiabatic cubic bodies at the center of a square cavity. They illustrated that the Nusselt number increases with an increase in the volume fraction of nanoparticles for most Rayleigh numbers. They also showed that the rate of heat transfer decreases as the body size increases at low Rayleigh numbers, and the opposite is true at high Rayleigh numbers.

Mixed convection of an $\mathrm{Al}_{2} \mathrm{O}_{3}$-water nanofluid in a cavity with a hot moving bottom wall and cold right, left, and top walls was investigated numerically by Mahmoodi [23]. The effect of variable properties of the nanofluid on mixed convection in a rectangular cavity was analyzed by Mazrouei Sebdani et al. [24].

Motivated by the investigations mentioned above, the purpose of the present work is to consider mixed convection flows of an $\mathrm{Al}_{2} \mathrm{O}_{3}$-water nanofluid in a square cavity with an inside heated obstacle and a moving upper lid that moves uniformly in the horizontal plane. The effects of the Richardson number and the diameter and volume fraction of $\mathrm{Al}_{2} \mathrm{O}_{3}$ nanoparticles on the flow, thermal fields, and heat transfer inside the cavity are studied.

\section{PHYSICAL MODELING AND GOVERNING EQUATIONS}

Figure 1 displays a two-dimensional lid-driven square cavity with an inside heated obstacle. The height and width of the square cavity are denoted by $L$. The cavity is filled by a suspension of $\mathrm{Al}_{2} \mathrm{O}_{3}$ nanoparticles in water. The bottom and vertical walls are insulated, whereas the top moving wall is kept at a low temperature $T_{c}$. In order to induce the buoyancy effect, an obstacle with a relatively higher temperature $T_{h}$ is located on the bottom wall of the cavity.

The nanofluid in the enclosure is assumed to be Newtonian, incompressible, and laminar. In addition, it is assumed that both the fluid phase and nanoparticles are in the thermal equilibrium state and move with the same velocity. The momentum equation is written in the Boussinesq approximation. The thermophysical properties of nanoparticles and the water as the base fluid at $T=25^{\circ} \mathrm{C}$ are presented in Table $1\left(c_{p}\right.$ is the heat capacity, $\rho$ is the density, $K$ is the thermal conductivity, $\beta$ is the thermal expansion coefficient, and $\mu$ is the dynamic viscosity). 
Table 1. Thermophysical properties of water and nanoparticles at $T=25^{\circ} \mathrm{C}$

\begin{tabular}{c|c|c|c|c|c}
\hline $\begin{array}{c}\text { Nanofluid } \\
\text { component }\end{array}$ & $\begin{array}{c}c_{p}, \\
\mathrm{~J} /(\mathrm{kg} \cdot \mathrm{K})\end{array}$ & $\begin{array}{c}\rho, \\
\mathrm{kg} / \mathrm{m}^{3}\end{array}$ & $\begin{array}{c}K, \\
\mathrm{~W} /(\mathrm{m} \cdot \mathrm{K})\end{array}$ & $\begin{array}{c}\beta \cdot 10^{-5}, \\
\mathrm{~K}^{-1}\end{array}$ & $\begin{array}{c}\mu \cdot 10^{-4}, \\
\mathrm{~kg} /(\mathrm{m} \cdot \mathrm{s})\end{array}$ \\
\hline $\begin{array}{c}\text { Fluid phase (water) } \\
\text { Solid phase }\left(\mathrm{Al}_{2} \mathrm{O}_{3}\right)\end{array}$ & $\begin{array}{r}479 \\
765\end{array}$ & $\begin{array}{r}997.1 \\
3970.0\end{array}$ & $\begin{array}{c}0.6 \\
25.0\end{array}$ & $\begin{array}{c}21.00 \\
0.85\end{array}$ & $\begin{array}{l}8.9 \\
-\end{array}$ \\
\hline
\end{tabular}

The thermal conductivity and viscosity of the nanofluid are taken into consideration as variable properties; both of them change with the volume fraction and temperature of nanoparticles. Under the above-made assumptions, the system of governing equations has the form

$$
\begin{gathered}
\frac{\partial u}{\partial x}+\frac{\partial v}{\partial y}=0, \\
u \frac{\partial u}{\partial x}+v \frac{\partial u}{\partial y}=-\frac{1}{\rho_{n f}} \frac{\partial p}{\partial x}+\nu_{n f} \nabla^{2} u, \\
u \frac{\partial v}{\partial x}+v \frac{\partial v}{\partial y}=-\frac{1}{\rho_{n f}} \frac{\partial p}{\partial y}+\nu_{n f} \nabla^{2} v+\frac{(\rho \beta)_{n f}}{\rho_{n f}} g \Delta T, \\
u \frac{\partial T}{\partial x}+v \frac{\partial T}{\partial y}=\alpha_{n f} \nabla^{2} T,
\end{gathered}
$$

where $\rho_{n f}, \nu_{n f}$, and $\alpha_{n f}$ are the density, kinematic viscosity, and thermal diffusivity of the nanofluid, respectively.

The dimensionless parameters may be presented as

$$
X=\frac{x}{L}, Y=\frac{y}{L}, V=\frac{v}{u_{0}}, U=\frac{u}{u_{0}}, \Delta T=T_{h}-T_{c}, \theta=\frac{T-T_{c}}{\Delta T}, \quad P=\frac{p}{\rho_{n f} u_{0}^{2}} .
$$

The velocity scale in passing to the dimensionless variables is the lid velocity $u_{0}$. Hence, the similarity criteria have the form

$$
\operatorname{Re}=\frac{\rho_{f} u_{0} L}{\mu_{f}}, \quad \operatorname{Ri}=\frac{\operatorname{Ra}}{\operatorname{Pr}^{2} \operatorname{Re}^{2}}, \quad \operatorname{Ra}=\frac{g \beta_{f} \Delta T L^{3}}{\nu_{f} \alpha_{f}}, \quad \operatorname{Pr}=\frac{\nu_{f}}{\alpha_{f}} .
$$

The dimensionless form of the governing equations (1) is

$$
\begin{gathered}
\frac{\partial U}{\partial X}+\frac{\partial V}{\partial Y}=0 \\
U \frac{\partial U}{\partial X}+V \frac{\partial U}{\partial Y}=-\frac{\partial P}{\partial X}+\frac{\nu_{n f}}{\nu_{f}} \frac{1}{\operatorname{Re}} \nabla^{2} U, \\
U \frac{\partial V}{\partial X}+V \frac{\partial V}{\partial Y}=-\frac{\partial P}{\partial Y}+\frac{\nu_{n f}}{\nu_{f}} \frac{1}{\operatorname{Re}} \nabla^{2} V+\frac{\operatorname{Ri}}{\operatorname{Pr}} \frac{\beta_{n f}}{\beta_{f}} \Delta \theta, \\
U \frac{\partial \theta}{\partial X}+V \frac{\partial \theta}{\partial Y}=\frac{\alpha_{n f}}{\alpha_{f}} \nabla^{2} \theta .
\end{gathered}
$$

The boundary conditions are:

$$
\begin{aligned}
& \partial \theta / \partial X=0 \text {, and } U=V=0 \text { on the left wall; } \\
& \partial \theta / \partial X=0 \text { and } U=V=0 \text { on the right wall; } \\
& \partial \theta / \partial Y=0 \text { and } U=V=0 \text { on the bottom wall; } \\
& \theta=0, U=1 \text {, and } V=0 \text { on the top wall; } \\
& \theta=1, U=0 \text {, and } V=0 \text { on the obstacle walls. }
\end{aligned}
$$

The thermal diffusivity and effective density of the nanofluid are

$$
\alpha_{n f}=k_{n f} /\left(\rho c_{p}\right)_{n f}, \quad \rho_{n f}=\varphi \rho_{s}+(1-\varphi) \rho_{f},
$$

where $\varphi$ is the volume fraction of nanoparticles in the fluid. 
The heat capacity $\left(\rho c_{p}\right)_{n f}$ and thermal expansion coefficient $(\rho \beta)_{n f}$ of the nanofluid are

$$
\left(\rho c_{p}\right)_{n f}=\varphi\left(\rho c_{p}\right)_{s}+(1-\varphi)\left(\rho c_{p}\right)_{f}, \quad(\rho \beta)_{n f}=\varphi(\rho \beta)_{s}+(1-\varphi)(\rho \beta)_{f} .
$$

The effective viscosity of the nanofluid is calculated as

$$
\mu_{\text {eff }}=\mu_{f}(1+2.5 \varphi)\left[1+\eta\left(d_{p} / L\right)^{-2 \varepsilon} \varphi^{2 / 3}(\varepsilon+1)\right],
$$

where $d_{p}$ is the nanoparticle diameter. This well-validated model was presented by Jang et al. [25] for a fluid containing a dilute suspension of small rigid spherical particles. The empirical constants $\varepsilon$ and $\eta$ for $\mathrm{Al}_{2} \mathrm{O}_{3}$ are 0.25 and 280 , respectively.

It is worth mentioning that the viscosity of the base fluid (water) is considered to vary with temperature; therefore, the viscosity of water is calculated as

$$
\mu_{\mathrm{H}_{2} \mathrm{O}}=\left(1.2723 T_{r c}^{5}-8.736 T_{r c}^{4}+33.708 T_{r c}^{3}-246.6 T_{r c}^{2}+518.78 T_{r c}+1153.9\right) \cdot 10^{6},
$$

where $T_{r c}=\log (T-273)$.

The effective thermal conductivity of nanoparticles in a stationary liquid is calculated by the HamiltonCrosser (H-C) model [26]

$$
\frac{k_{s t}}{k_{f}}=\frac{k_{s}+2 k_{f}-2 \varphi\left(k_{f}-k_{s}\right)}{k_{s}+2 k_{f}+\varphi\left(k_{f}-k_{s}\right)}
$$

where $k_{s}$ is the thermal conductivity of the disperse solid nanoparticles.

The total dimensionless thermal conductivity of the nanofluid is

$$
\frac{k_{n f}}{k_{f}}=\frac{k_{s t}}{k_{f}}+\frac{k_{c}}{k_{f}}=\frac{k_{s}+2 k_{f}-2 \varphi\left(k_{f}-k_{s}\right)}{k_{s}+2 k_{f}+\varphi\left(k_{f}-k_{s}\right)}+c \frac{\mathrm{Nu}_{p} d_{f}\left(2-D_{f}\right) D_{f}}{\operatorname{Pr}\left(1-D_{f}\right)^{2}} \frac{\left[\left(d_{\max } / d_{\min }\right)^{1-D_{f}}-1\right]^{2}}{\left(d_{\max } / d_{\min }\right)^{2-D_{f}}-1} \frac{1}{d_{p}} .
$$

This model was proposed by Xu et al. [27], and it was chosen in this study to describe the thermal conductivity of nanofluids. Here $c$ is an empirical constant (e.g., $c=85$ for deionized water and $c=280$ for ethylene glycol), which is independent of the presence of nanoparticles, $\mathrm{Nu}_{p}$ is the Nusselt number for the fluid flowing around a spherical particle $\left(\mathrm{Nu}_{p}=2\right.$ for a single particle in this work). The fluid (water) molecule diameter is $d_{f}=4.5 \cdot 10^{-10} \mathrm{~m}$. The fractal dimension $D_{f}$ is determined by

$$
D_{f}=2-\ln (\varphi) / \ln \left(d_{p, \min } / d_{p, \max }\right),
$$

where $d_{p, \max }$ and $d_{p, \text { min }}$ are the maximum and minimum diameters of nanoparticles, respectively:

$$
d_{p, \max }=d_{p} \frac{D_{f}-1}{D_{f}}\left(\frac{d_{p, \min }}{d_{p, \max }}\right)^{-1}, \quad d_{p, \min }=d_{p} \frac{D_{f}-1}{D_{f}} .
$$

The Nusselt number based on the cavity width is evaluated as

$$
\mathrm{Nu}=h_{n f} L / k_{f},
$$

where

$$
h_{n f}=\frac{q}{T_{h}-T_{c}}, \quad q=-\left.k_{n f} \frac{T_{h}-T_{c}}{L} \frac{\partial \theta}{\partial Y}\right|_{Y=1} .
$$

Substituting Eqs. (3) into Eq. (2) yields the following equation for the Nusselt number:

$$
\mathrm{Nu}=-\left.\frac{k_{n f}}{k_{f}} \frac{\partial \theta}{\partial Y}\right|_{Y=1} .
$$

The average Nusselt number is obtained by integrating Eq. (4) along the top wall.

\section{NUMERICAL METHOD}

The governing equations for continuity, momentum, and energy with the boundary conditions in this investigation were calculated numerically based on the finite volume method on a staggered grid system. The SIMPLE algorithm is used to solve the coupled system of the governing equations. The convection terms are approximated by a hybrid scheme, which is conducive to a stable solution. In addition, a second-order central difference scheme 446 


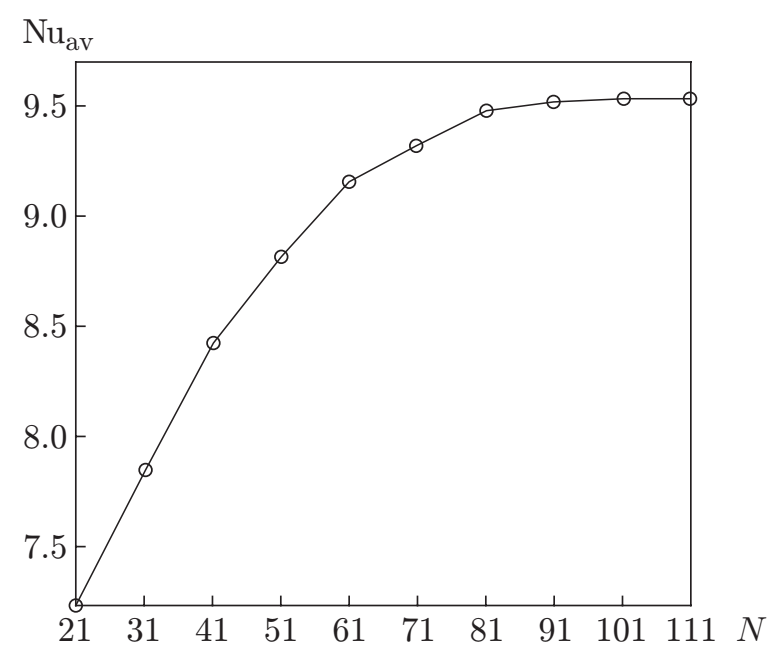

Fig. 2. Grid convergence for the average Nusselt number.

Table 2. Comparisons of results of cavity flow calculations by different models

\begin{tabular}{l|c|c|c|c|c|c}
\hline \multicolumn{1}{c|}{ Model } & $\mathrm{Ra}$ & $u_{\max }$ & $Y$ & $v_{\max }$ & $X$ & $\mathrm{Nu}_{\mathrm{av}}$ \\
\hline Present study & & 16.05200 & 0.817 & 19.528 & 0.110 & 2.215 \\
Model of Lin and Violi [28] & \multirow{2}{*}{$10^{4}$} & 16.15800 & 0.819 & 19.648 & 0.112 & 2.243 \\
Model of Towari and Das [29] & & 16.14390 & 0.822 & 19.665 & 0.110 & 2.195 \\
Model of Hadjisophocleous et al. [30] & & 15.99500 & 0.814 & 18.894 & 0.103 & 2.290 \\
\hline Present study & & 36.81200 & 0.856 & 68.7910 & 0.06200 & 4.517 \\
Model of Lin and Violi [28] & \multirow{2}{*}{$10^{5}$} & 36.73200 & 0.858 & 68.2880 & 0.06300 & 4.511 \\
Model of Towari and Das [29] & & 34.30000 & 0.856 & 68.7646 & 0.05935 & 4.450 \\
Model of Hadjisophocleous et al. [30] & & 37.14400 & 0.855 & 68.9100 & 0.06100 & 4.964 \\
\hline Present study & & 66.44500 & 0.87300 & 221.74800 & 0.03980 & 8.795000 \\
Model of Lin and Violi [28] & \multirow{3}{*}{$10^{6}$} & 66.46987 & 0.86851 & 222.33950 & 0.03804 & 8.757933 \\
Model of Towari and Das [29] & & 65.58660 & 0.83900 & 219.73610 & 0.04237 & 8.803000 \\
Model of Hadjisophocleous et al. [30] & & 66.42000 & 0.89700 & 226.40000 & 0.02060 & 10.390000 \\
\hline
\end{tabular}

is utilized for the diffusion terms. The algebraic system resulting from numerical discretization is calculated by utilizing the TDMA method.

To verify grid independence, numerical calculations were performed for several mesh sizes. The average Nusselt number on the hot body wall at $d=0.2 L, \mathrm{Ri}=1, h=0.1 L, d p=40 \mathrm{~nm}, T=320 \mathrm{~K}$, and $\varphi=0.03$ is obtained for different grid sizes (see Fig. 2, where $N$ is the number of grid nodes in the $x$ direction). As can be observed, a $101 \times 101$ uniform grid yields the required accuracy; hence, it is applied for all simulation exercises in this work.

To ensure the accuracy and validity of this new model, we analyze a square cavity filled by the base fluid with $\operatorname{Pr}=0.7$ and different Rayleigh numbers (Ra). Table 2 shows the comparison between the results obtained with the new model and the values presented in the literature [28-30]. The quantitative comparisons for the average Nusselt numbers indicate that they are in excellent agreement.

\section{RESULTS AND DISCUSSION}

The thermal behavior and flow characteristics in a square cavity filled by a nanofluid, which has a moving upper lid and a square hot obstacle, was investigated by means of numerical simulations based on the finite volume method. The influence of parameters such as the nanofluid solid volume fraction, nanoparticle diameter, and Richardson number on streamlines and isotherms was also studied. The moving upper lid of the cavity was preserved at a low temperature, while the other walls were assumed to be thermally insulated. The buoyancy force resulting 
from the temperature difference between the obstacle and the hot wall and the shear force resulting from the upper lid motion caused flow generation and heat transfer in the cavity. It should be noted that the numerical simulations in the present study were undertaken by varying the Richardson number Ri with the Grashof number being fixed at $\mathrm{Gr}=10^{4}$.

Figure 3 displays the streamlines and isotherms at $T=320 \mathrm{~K}, h=0.2 L, \varphi=0.05, \mathrm{Ri}=1, d=0.2 L$, and different values of the diameter of nanoparticles dispersed in water. In this case, the streamlines show the formation of a clockwise central primary (vortex) cell above the obstacle, which occupies a vast area of the cavity. Moreover, a small vortex is formed in the space between the obstacle and the left adiabatic wall. The isotherms are also intensified in areas around the isothermal walls, while the distribution of these lines is very sparse in the central areas of the cavity.

With increasing nanoparticle diameter, no meaningful change happens in the flow pattern in the cavity, but the intensity of the primary cell slightly deceases. An increase in the nanoparticle diameter causes a decrease in the intensity of the isotherms near the hot walls, which indicates a decrease in the temperature gradient. It is, therefore, expected that heat transfer slightly decreases in the cavity with increasing nanoparticle diameter.

Figure 4 shows the streamlines and isotherms at $d_{p}=20 \mathrm{~nm}, \mathrm{Ri}=1, T=320 \mathrm{~K}, h=0.2 L, d=0.2 L$, and different solid volume fractions. The formation of a clockwise central cell and a small vortex in the cavity can be seen. Paying close attention to the streamlines, one could infer that an increase in the solid volume fraction results in a decrease in the severity and strength of the central cell, but no particular changes in the flow pattern. The isotherms are distributed intensely near the hot walls, but negligibly in the central areas of the cavity. An increase in the solid volume fraction causes a slight decrease in the intensity of the isotherms near nonadiabatic surfaces. A decrease in the temperature gradient with increasing solid volume fraction is a result of an increase in the thermal conductivity of the fluid. Heat transfer in the cavity cannot be predicted by the isotherms because it depends on the temperature gradient and thermal conductivity of the fluid, which, in turn, depend on the solid volume fraction. Therefore, heat transfer should be determined on the basis of the Nusselt diagrams.

Figure 5 displays the streamlines and isotherms at $d=0.2 L, d_{p}=20 \mathrm{~nm}, h=0.2 L, \varphi=0.05, T=320 \mathrm{~K}$, and different Richardson numbers. At Ri $=0.01$, with perfect dominance of the shear force over the buoyancy force, the central cell is formed owing to the upper lid motion. Moreover, two small vortices are formed on the two sides of the hot obstacle. As it is clear from Fig. 5, the streamlines are very intense near the moving lid, which indicates a very high velocity of the fluid flow in these areas. At $\mathrm{Ri}=0.01$, severe intensity of the isotherms and the formation of the thermal boundary layer close to the hot walls are observed. A very high temperature gradient indicates high heat transfer in the cavity in this position. At $\mathrm{Ri}=1$, the effect of the buoyancy force increases, and the central cell, therefore, widens and occupies a larger space in the cavity. The isotherms display a decrease in intensity close to the hot walls and a decrease in the temperature gradient. In this case, the heat transfer rate is appreciably smaller than that at $\mathrm{Ri}=0.01$. As the Richardson number is increased up to $\mathrm{Ri}=100$, the buoyancy force dominates over the shear force, and the primary cell is formed in the center of the cavity. As it follows from Fig. 5, with increasing Richardson number, the primary cell separates from the upper lid and settles in the central area of the cavity, which indicates a decrease in the effect of the upper lid motion on the cell. The isotherms also show a severe decrease in the temperature gradient as compared to the cases with $\mathrm{Ri}=0.01$ and 1.00 . It is, therefore, expected that the heat transfer rate decreases in the cavity with increasing Richardson number. It needs to be mentioned that the buoyancy and shear forces act in the same direction and reinforce each other in all cases considered in this paper.

Figure 6 displays the average Nusselt as a function of the Richardson number for different nanoparticle diameters. As the diagram vividly shows, the heat transfer rate increases with decreasing nanoparticle diameter. The increase in the Nusselt number is approximately $11.4 \%$ as the nanoparticle diameter is increased in the interval of $20-30 \mathrm{~nm}$.

Figure 7 displays the Nusselt number as a function of the Richardson number for different solid volume fractions. An increase in the Nusselt number and, accordingly, an increase in the heat transfer rate in the cavity with increasing solid volume fraction are crystal clear in the diagram. Additionally, heat transfer displays more upsurges at higher Richardson numbers as compared to lower Richardson number values. 
(a)

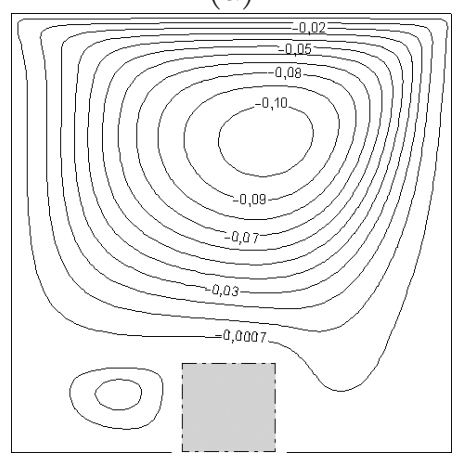

(c)

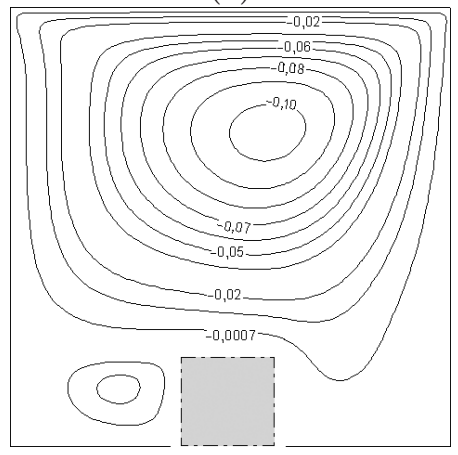

(e)

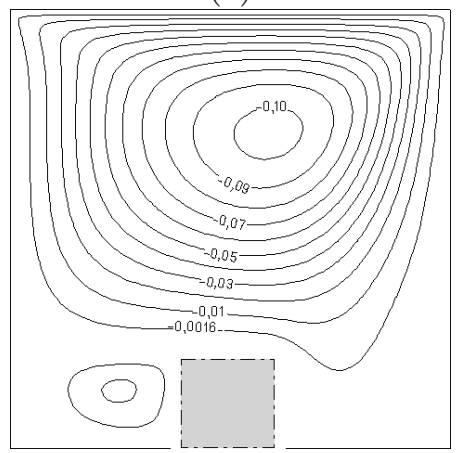

(g)

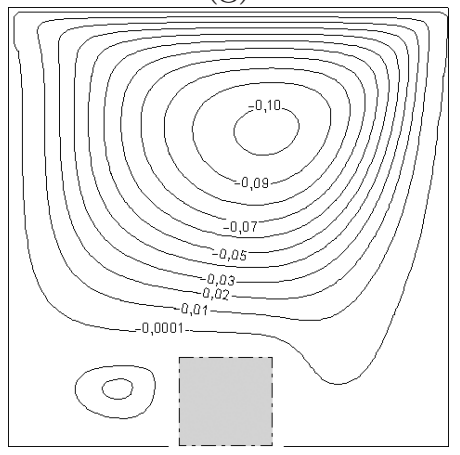

(b)

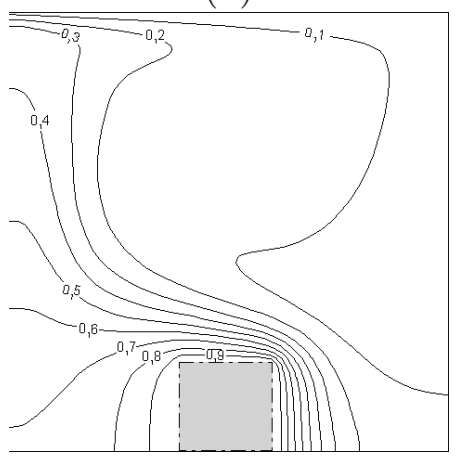

(d)

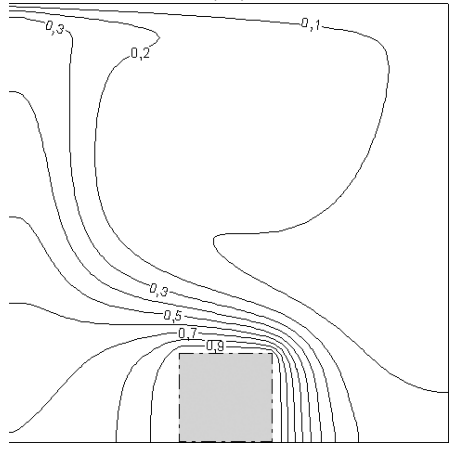

(f)

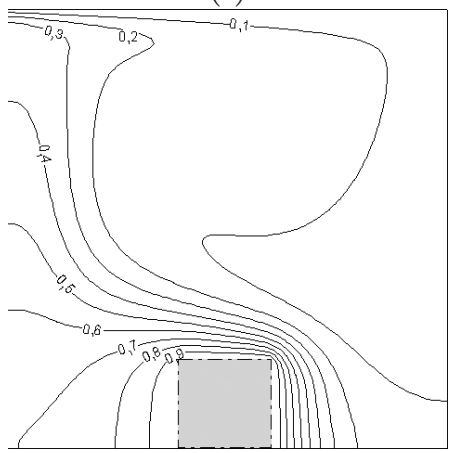

(h)

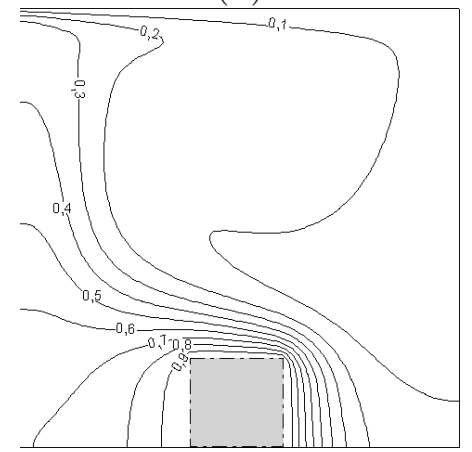

Fig. 3. Streamlines (a, c, e, and g) and isotherms (b, d, f, and h) at $T=320 \mathrm{~K}, h=0.2 L, \varphi=0.05, \mathrm{Ri}=1$, $d=0.2 L$, and different nanoparticle diameters: $d_{p}=20$ ( $\mathrm{a}$ and b), 40 (c and d), 60 (e and f), and $80 \mathrm{~nm}(\mathrm{~g}$ and $\mathrm{h}$ ). 
(a)

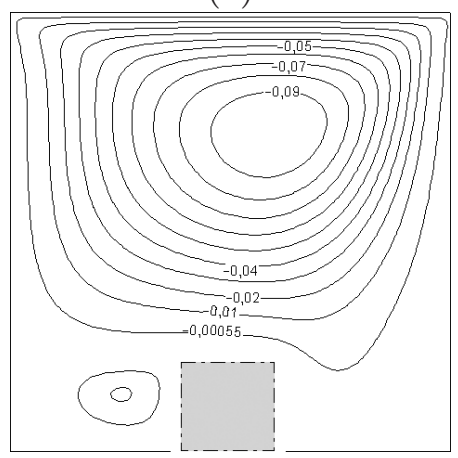

(c)

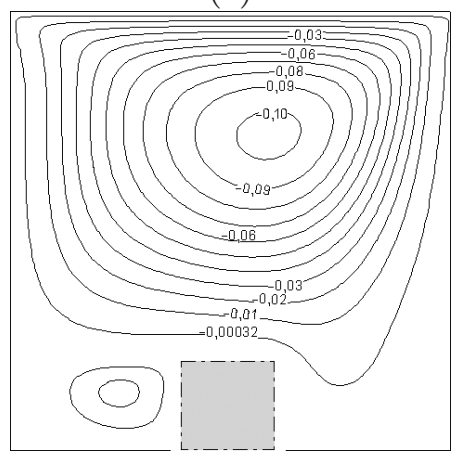

(e)

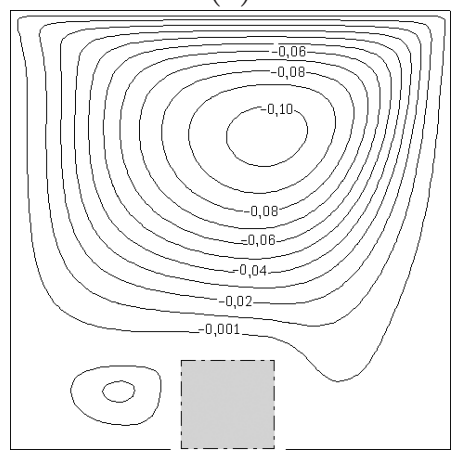

(g)

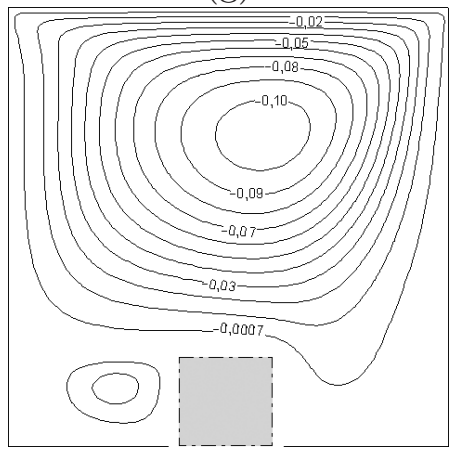

(b)

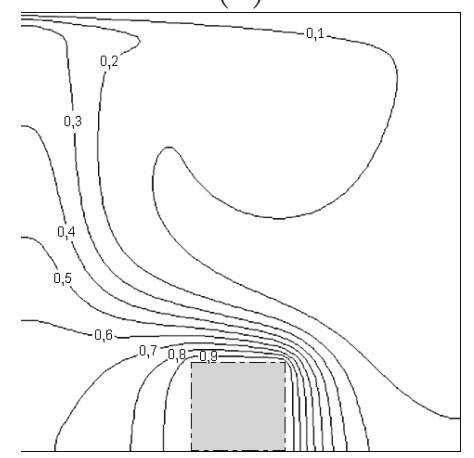

(d)

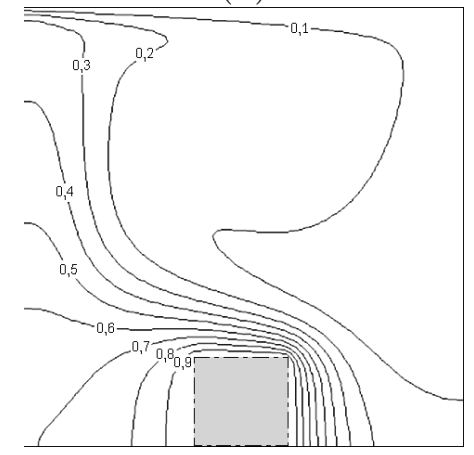

(f)

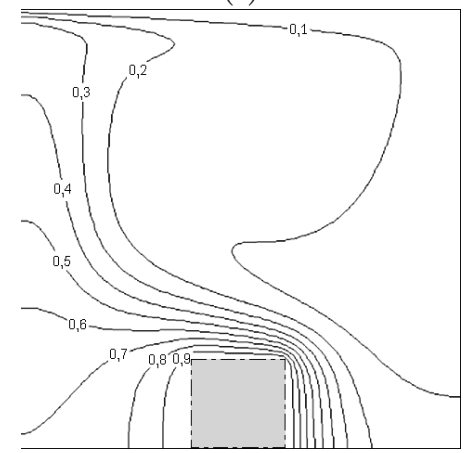

(h)

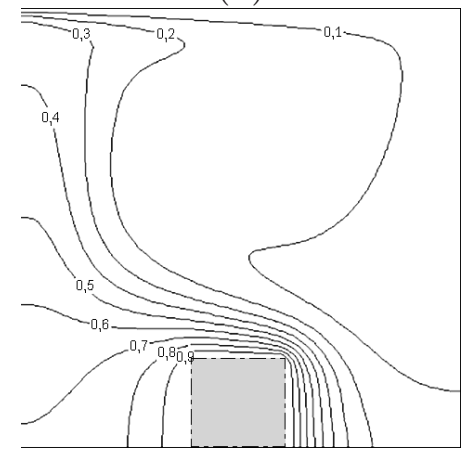

Fig. 4. Streamlines (a, c, e, and g) and isotherms (b, d, f, and h) at $d_{p}=20 \mathrm{~nm}, \mathrm{Ri}=1, T=320 \mathrm{~K}, h=0.2 L$, $d=0.2 L$, and different solid volume fractions: $\varphi=0$ (a and b), 0.01 (c and d), 0.03 (e and f), and 0.05 ( $\mathrm{g}$ and $\mathrm{h}$ ). 
(a)

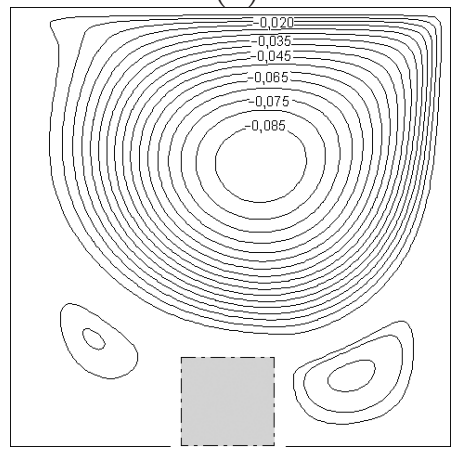

(c)

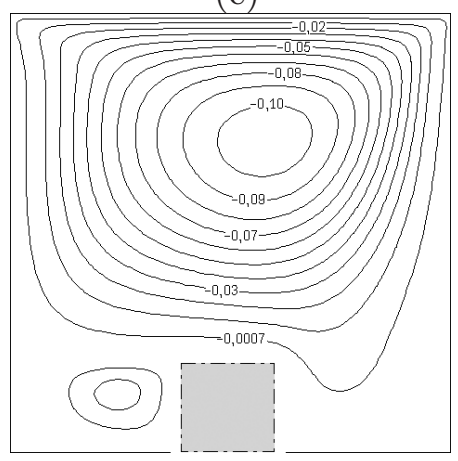

(e)

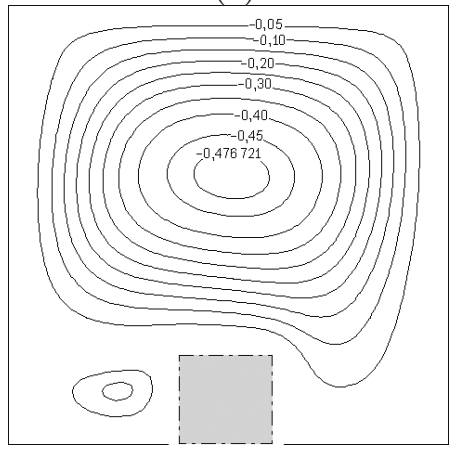

(b)

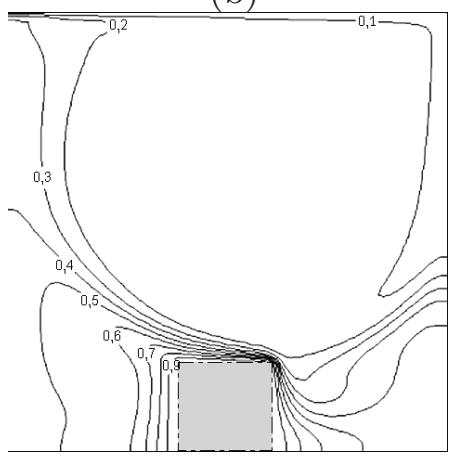

(d)

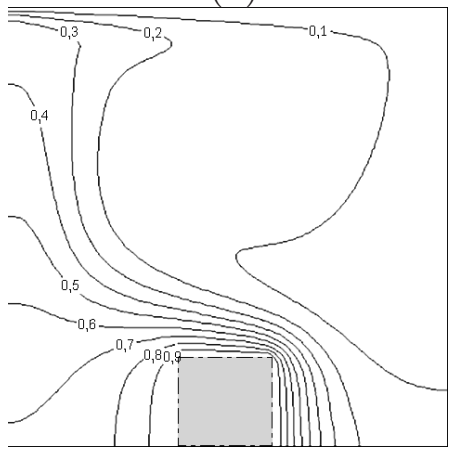

(f)

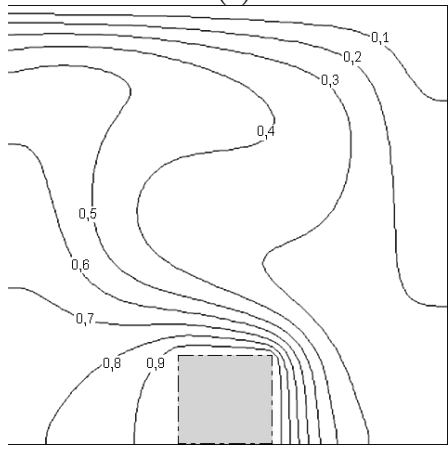

Fig. 5. Streamlines (a, c, and e) and isotherms (b, d, and f) at $d=0.2 L, d_{p}=20 \mathrm{~nm}$, $h=0.2 L, \varphi=0.05, T=320 \mathrm{~K}$, and different Richardson numbers: $\mathrm{Ri}=0.01$ (a and $\mathrm{b}$ ), 1 (c and d), and 100 (e and f).

\section{CONCLUSIONS}

In this paper, the effect of variable properties of an $\mathrm{Al}_{2} \mathrm{O}_{3}$-water nanofluid on mixed convection heat transfer and fluid flow in a two-dimensional lid-driven square cavity with an inside hot obstacle on the bottom wall was studied numerically.

For all Richardson numbers and volume fractions of nanoparticles, it is observed that the nanofluid descends downward along the right wall, moves horizontally above the obstacle to the left corner of the cavity along the bottom wall, and then moves upward and forms an eddy inside the cavity. As the Richardson number is increased, the clockwise rotating vortex becomes larger due to influence of the hot obstacle.

If the Richardson number and the solid volume fraction are kept constant and the nanoparticle diameter is varied from 20 to $80 \mathrm{~nm}$, there are only minor changes in the flow pattern and isotherms inside the cavity.

The average Nusselt number decreases as the Richardson number increases. 


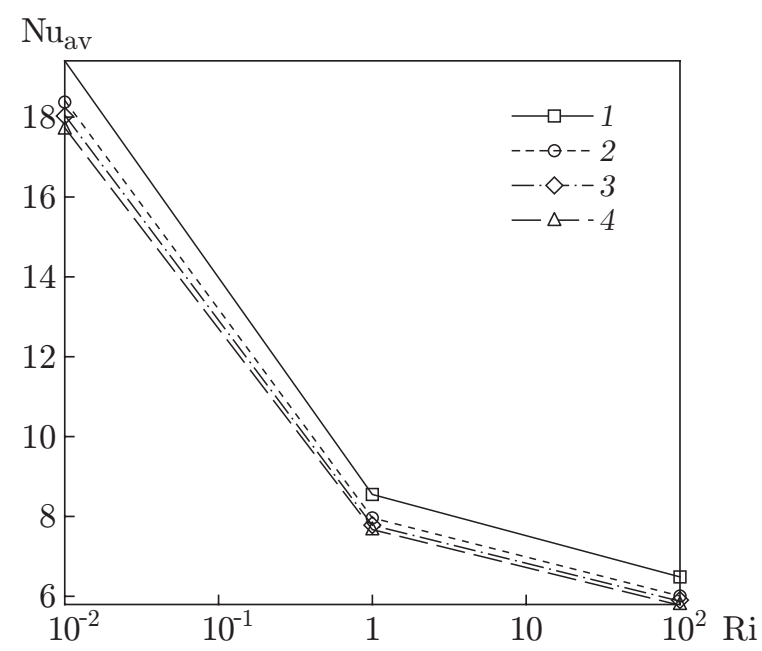

Fig. 6.

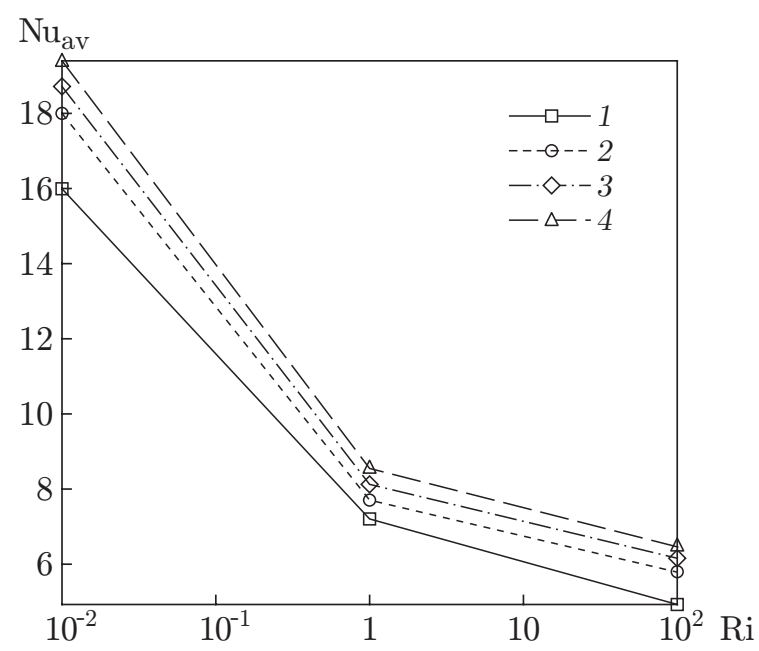

Fig. 7.

Fig. 6. Nusselt number versus the Richardson number at $\varphi=0.05, T=320 \mathrm{~K}, h=0.2 L$, and different nanoparticle diameters: $d_{p}=20(1), 40(2), 60(3)$, and $80 \mathrm{~nm}(4)$.

Fig. 7. Nusselt number versus the Richardson number at $d_{p}=20 \mathrm{~nm}, h=0.2 L, T=320 \mathrm{~K}$, and different volume fractions of nanoparticles: $\varphi=0(1), 0.01(2), 0.03(3)$, and $0.05(4)$.

Addition of $\mathrm{Al}_{2} \mathrm{O}_{3}$ nanoparticles produces a remarkable enhancement of heat transfer as compared to that of the pure fluid.

For all Richardson numbers considered in this study, the flow velocity and, correspondingly, the heat transfer rate decrease as the mean nanoparticle diameter increases.

The authors would like to acknowledge Dr. S. Saedodin, Mr. H. Hajmohammad, and S. H. Rostamian for useful discussions of the present paper.

\section{REFERENCES}

1. S. U. S. Choi, "Enhancing Thermal Conductivity of Fluids with Nanoparticles," Developments and Applications of Non-Newtonian Flows, Ed. by D. A. Siginer, and H. P. Wang (ASME, New York, 1995), FED-V. 231/MDV. 66, pp. 99-105.

2. Y. Xuan and Q. Li, "Investigation on Convective Heat Transfer and Flow Features of Nanofluids," J. Heat Transfer 125, 151-155 (2003).

3. S. Lee, S. U. S. Choi, S. Li, and J. A. Eastman, "Measuring Thermal Conductivity of Fluids Containing Oxide Nanoparticles," Int. J. Heat Mass Transfer 121, 280-289 (2001).

4. H. Q. Xie, J. C. Wang, T. G. Xi, et al., "Dependence of the Thermal Conductivity of Nanoparticle-Fluid Mixture on the Base Fluid," J. Mater. Sci. Lett. 21, 1469-1471 (2002).

5. H. E. Patel, T. Pradeep, T. Sundararajan, et al., "A Micro Convection Model for Thermal Conductivity of Nanofluid," Pramana-J. Phys. 65, 863-869 (2005).

6. H. Chang, C. S. Jwo, C. H. Lo, et al., "Rheology of CuO Nanoparticle Suspension Prepared by ASNSS," Rev. Adv. Mater. Sci. 10, 128-132 (2005).

7. J. Imberger and P. F. Hamblin, "Dynamics of Lakes, Reservoirs, and Cooling Ponds," Annual Rev. Fluid Mech. 14, 153-187 (1982).

8. M. K. Moallemi and K. S. Jang, "Prandtl Number Effects on Laminar Mixed Convection Heat Transfer in a Lid-Driven Cavity," Int. J. Heat Mass Transfer 35, 1881-1892 (1992).

9. C. K. Cha and Y. Jaluria, "Recirculating Mixed Convection Flow for Energy Extraction," Int. J. Heat Mass Transfer 27, 1801-1810 (1984).

10. F. J. K. Ideriah, "Prediction of Turbulent Cavity Flow Driven by Buoyancy and Shear," J. Mech. Eng. Sci. 22, 287-295 (1980). 
11. L. A. B. Pilkington, "Review Lecture: The Float Glass Process," Proc. Roy. Soc. London, Ser. A 314, 1-25 (1969).

12. F. Talebi, A. H. Mahmoudi, and M. Shahi, "Numerical Study of Mixed Convection Flows in a Square Lid-Driven Cavity Utilizing Nanofluid," Int. Comm. Heat Mass 37, 79-90 (2010).

13. E. Abu-Nada and A. J. Chamkha, "Mixed Convection Flow in a Lid Driven Square Enclosure Filled with a Nanofluid," Eur. J. Mech. B. Fluid 29, 472-482 (2010).

14. M. Mahmoodi, "Mixed Convection Inside Nanofluid Filled Rectangular Enclosures with Moving Bottom Wall," Thermal Sci. 15, 889-903 (2011).

15. A. Arefmanesh and M. Mahmoodi, "Effects of Uncertainties of Viscosity Models for $\mathrm{Al}_{2} \mathrm{O}_{3}$-Water Nanofluid on Mixed Convection Numerical Simulations," Int. J. Thermal Sci. 50, 1706-1719 (2011).

16. Hemmat M. Esfe, F. Ghadak, A. Haghiri, and S. Mirtalebi, "Numerical Study of Mixed Convection Flows in a Two-Sided Inclined Lid-Driven Cavity Utilizing Nano-Fluid with Various Inclination Angles and Ununiformed Temperature," Aerospace Mech. J. 8 (2), 69-83 (2012).

17. A. Fereidoon, S. Saedodin, Hemmat M. Esfe, and M. J. Noroozi, "Evaluation of Mixed Convection in Inclined Square Lid Driven Cavity Filled with $\mathrm{Al}_{2} \mathrm{O}_{3}$ /Water Nanofluid," Eng. Appl. Comput. Fluid Mech. 7, 55-65 (2013).

18. H. Zarei, S. H. Rostamian, and Hemmat M. Esfe, "Heat Transfer Behavior of Mixed Convection Flow in Lid Driven Cavity Containing Hot Obstacle Subjected to Nanofluid with Variable Properties," J. Basic. Appl. Sci. Res. 3, 713-721 (2013).

19. A. Z. Ghadi, M. J. Noroozi, and Hemmat M. Esfe, "Nanofluid Implementation for Heat Transfer Augmentation of Magneto Hydrodynamic Flows in a Lid-Driven Cavity using Experimental-Based Correlations," Int. J. Appl. Electromagnetics Mech. 42 (4), 589-602 (2013).

20. M. R. Heidari, Hemmat M. Esfe, H. Hajmohammad, and M. Akbari, "Mixed Convection Heat Transfer in a Double Lid-Driven Inclined Square Enclosure Subjected to $\mathrm{Cu}$-Water Nanofluid with Particle Diameter of 90 nm," Heat Transfer Res. 45 (1), 75-95 (2014).

21. M. Nikfar and M. Mahmoodi, "Meshless Local Petrov - Galerkin Analysis of Free Convection of Nanofluid in a Cavity with Wavy Side Walls," Eng. Anal. Boundary Elements 36, 433-445 (2012).

22. M. Mahmoodi and S. Mazrouei Sebdani, "Natural Convection in a Square Cavity Containing a Nanofluid and an Adiabatic Square Block at the Center," Superlattices Microstruct. 52, 261-275 (2012).

23. M. Mahmoodi, "Mixed Convection Inside Nanofluid Filled Rectangular Enclosures with Moving Bottom Wall," Thermal Sci. 15 (3), 889-903 (2011).

24. Mazrouei S. Sebdani, M. Mahmoodi, and S. M. Hashemi, "Effect of Nanofluid Variable Properties on Mixed Convection in a Square Cavity," Int. J. Thermal Sci. 52, 112-126 (2012).

25. S. P. Jang, J. H. Lee, K. S. Hwang, and S. U. S. Choi, "Particle Concentration and Tube Size Dependence of Viscosities of $\mathrm{Al}_{2} \mathrm{O}_{3}$-Water Nanofluids Flowing Through Micro- and Minitubes," Appl. Phys. Lett. 91, 24-31 (2007).

26. R. L. Hamilton and O. K. Crosser, "Thermal Conductivity of Heterogeneous Two Component Systems," Indust. Eng. Chem. Fundam. 1, 187-191 (1962).

27. J. Xu, B. Yu, M. Zou, and P. Xu, "A New Model for Heat Conduction of Nanofluids Based on Fractal Distributions of Nanoparticles," J. Phys. D 39, 4486-4490 (2006).

28. K. C. Lin and A. Violi, "Natural Convection Heat Transfer of Nanofluids in a Vertical Cavity: Effects of NonUniform Particle Diameter and Temperature on Thermal Conductivity," Int. J. Heat Fluid Flow 31, 236-245 (2010).

29. R. K. Tiwari and M. K. Das, "Heat Transfer Augmentation in a Two-Sided Lid-Driven Differentially Heated Square Cavity Utilizing Nanofluids," Int. J. Heat Mass Transfer 50, 2002-2018 (2007).

30. G. V. Hadjisophocleous, A. C. M. Sousaand, and J. E. S. Venart, "Predicting the Transient Natural Convection in Enclosures of Arbitrary Geometry using a Nonorthogonal Numerical Model," Numer. Heat Transfer. A 13, 373-392 (1998). 